SPECIAL ISSUE PAPER

\title{
Training Paediatric Therapists to Deliver Constraint- Induced Movement Therapy (CIMT) in Sub-Saharan Africa
}

\author{
Patty Coker-Bolt ${ }^{1 * \dagger}$, Stephanie C. DeLuca ${ }^{2} \&$ Sharon L. Ramey ${ }^{2}$ \\ ${ }^{1}$ Medical University of South Carolina, Charleston, SC, USA \\ ${ }^{2}$ Virginia Tech Carilion Research Institute, Roanoke, VA, USA
}

\begin{abstract}
Hospitals and therapists in developing countries often seek to learn how to deliver new forms of evidenced-based practice (EBP), including paediatric constraint-induced movement therapy (CIMT). This study examines a partnership implemented in Ethiopia, which trained therapists in CIMT and proposes a framework for sustainable EBP training.

The aim of this study is to apply a translational and implementation framework to build capacity for CIMT in Addis Ababa, Ethiopia, that included intensive in-country training and hands-on delivery with patients, followed by clinical implementation and feedback. A goal was to develop a locally feasible, culturally relevant form of CIMT.

We framed our partnership model in terms of an implementation science model for therapists from multiple hospitals in Addis Ababa. Measures included workshop attendance, delivery of the curriculum and assessment of therapist's knowledge, skills and feedback postworkshop.

We established a successful partnership with a lead hospital and completed training for 12 therapists from five hospitals who demonstrated increases in knowledge and skills following training. We developed a new, practically useful, culturally appropriate form of CIMT for later implementation.

This partnership was limited to training of paediatric therapists in sub-Saharan Africa.

Future studies will report on therapists' ability to integrate this EBP training into clinical practice as well as future training. Copyright $\odot 2015$ John Wiley \& Sons, Ltd.
\end{abstract}

Received 18 January 2015; Revised 23 March 2015; Accepted 1 April 2015

\section{Keywords}

evidenced-based practice; international partnerships; implementation science; rehabilitation; constraint-induced movement therapy

\section{*Correspondence}

Patty Coker-Bolt, Division of Occupational Therapy, Medical University of South Carolina, 151 Rutledge Ave, Bldg B, MSC 962, Charleston 29439, SC, USA.

†Email: cokerpc@musc.edu

Published online 25 May 2015 in Wiley Online Library (wileyonlinelibrary.com) DOI: 10.1002/oti.1392

\section{Introduction}

Evidence-based practice (EBP) is defined as the integration of best research evidence, clinical expertise and patient values (Schreiber and Stern, 2005) and is critically important to all therapy interventions. Within the international rehabilitation community, many organizations have called for better training of therapists in diverse cultures and communities to implement the identified types of EBPs (e.g. Dawes et al., 2005; Dizon et al., 2012). Yet, a paucity of research provides 
guidelines or models for effective dissemination of new practice knowledge and evaluation of its impact on therapists' implementation of EBP, particularly specific therapies that require both new knowledge and skills. The challenge of implementing EBP is markedly elevated in settings with very low and scattered health service resources, such as sub-Saharan Africa.

The World Health Organization estimates that up to $7 \%$ of the population in developing countries has a moderate to severe disability (World Health Organization, 2011). Therapists in these countries often receive minimal, if any, formal training in specialty practices, such as paediatric occupational therapy, and have limited availability of specialty centres and experts in newly emerging techniques that support EBP (Donald et al., 2014). Additionally, national networks of providers and formal requirements for continuing education and certification often are scarce or only partially enacted. Just as challenging is the reality that many new therapies with demonstrated high efficacy have been developed for and tested only in higher resource countries and thus may not readily be well-suited for use, without adaptation, to settings and cultures that are markedly different.

The difficulty of better integrating evidenced-based treatments into clinical practice is certainly not unique to developing countries, but is an increasingly urgent concern for worldwide health and improving functional outcomes for individuals with disabilities. The emerging field of implementation science is dedicated to conducting research on the factors that promote (vs impede) timely, high-fidelity and equitable application of proven treatments. Estabrooks and Glasgow (2006), among others, have proposed a systematic, multilevel-system approach. Specifically, they identified three essential topics to consider when seeking to translate an EBP into a new clinical setting: (1) the structure of the healthcare setting where the intervention will be delivered; (2) the critical elements of the new intervention (compared with current standards of care) and how the intervention can be tested (on a limited basis) so that stakeholders can see the potential for positive changes likely to result from implementing the new EBP; and (3) the importance of developing a strong partnership with key individuals and/or agencies to plan jointly for the translation of the new EBP into the clinical setting. The engagement of local partners sets the stage for having strong, well-informed advocacy for both the initial and then later sustained implementation of the new EBP.
Similarly, Dawes et al. (2005) provided recommendations from an international working group of rehabilitation therapists, emphasizing the need for healthcare professionals in developing countries to both understand the principles of EBP and then adjust specific efficacious treatments to their local context, taking culture and resources into consideration. Research on implementation of EBP in developing countries has consistently identified the following barriers: limited preservice and in-service training, lack of active support and supervision for therapists in specific types of EBP and minimal or no methods for measuring therapists' skills in these specific evidence-backed therapies (Grimshaw et al., 2012; Dizon, Dizon, Regino, \& Gabriel, 2014). Rather than viewing a commitment to EBP as a luxury or elite option available primarily in high-resource settings, we advance the idea that EBP implementation is particularly compelling for developing countries to maximize the strategic investment of limited personnel and equipment to yield the best possible patient outcomes at the lowest cost while simultaneously reducing waste of resources on treatments known to be less effective, ineffective and/or potentially harmful (e.g. Novak et al., 2013).

Idro, Newton, Kiguli and Kakooza-Mwesige (2010) reviewed the current rehabilitation approaches used in sub-Saharan African countries and concluded that therapy interventions routinely focus on the use of passive movement, manipulation techniques, hand or hip (orthopaedic) surgery and orthotics for the hand (i.e. contracture management) - none of which meet the criteria for EBP for most children with neuromotor disabilities, including children with cerebral palsy (CP). More recently, in a landmark systematic review of all forms of treatment for children with CP, Novak et al. (2013) concluded that the previous treatments have either "low-level" or "inconclusive" evidence to support their continued use. (See the succeeding text for recommendations from Novak et al. regarding highly efficacious treatments). Independently, Donald et al. (2014) summarized the consensus of a working group of physicians who met to discuss the management of children with $\mathrm{CP}$ in Africa. This group determined that basic levels of care were greatly limited by poor availability of trained personnel with expertise in working with children with neuromotor disorders. They specified identified key training priorities, which included expansion of training for providers, which could occur through collaborations with overseas experts providing in-country education with a goal of later facilitating 
these local experts to provide training to others in their own country (Donald et al., 2014).

This paper provides an example of how we applied a translational and implementation framework to a partnership initiated by a medical hospital with paediatric researchers in the United States and then expanded to include four other hospitals and 12 therapists in Addis Ababa, Ethiopia. The overall aims of the partnership were to provide training to therapists in constraintinduced movement therapy (CIMT), the most strongly supported form of EBP for treating children with hemiparesis or asymmetric CP (cf. Novak et al., 2013; Ramey et al., 2013) and to establish ongoing supports for implementing and measuring the effects of delivering this new EBP, potentially creating expanded opportunities for future clinical research. We adopted a multilevel-system approach and collected descriptive indicators of the partnership, the training experience and the follow-up activities. In this paper, we describe the model we used, the training activities and indicators of impact, including objective measures, subjective evaluations and anecdotal and reflective perspectives. We received grant support for the specific aims to (1) a mutually acceptable plan for a training workshop in response to an enthusiastic invitation we received from an international healthcare entity that operated a major facility in Addis Ababa, Ethiopia; (2) provide in-person training in the use of CIMT, an EBP in which we had both clinical and scientific expertise (cf. Ramey et al., 2013), recognizing in advance the likely need to adapt the CIMT clinical protocol to local culture, resources and patient profiles; and (3) conduct a reflective evaluation to assess the extent to which the plan and model we used achieved our intended goals of producing qualified CIMT therapists who, in turn, would be able to provide CIMT to their patients and obtain clinical outcome data needed to justify offering this form of EBP to all eligible children on a sustained basis.

\section{Methods}

This process included engagement of physical therapists, occupational therapists, administrators and staff from five local, independent hospitals in Addis Ababa, Ethiopia. We completed the in-country training and first assessment in December of 2013 with a planned follow-up visit focused on clinical implementation and case review in May of 2014, and sent supplies and had communication in between the visits. The authors of this paper, an occupational therapist and rehabilitation and developmental scientists who developed and tested a high-intensity form of paediatric CIMT (P-CIMT) in the United States (Ramey et al., 2013), planned and led the training. The lead universities were the Medical University of South Carolina (MUSC) and the Virginia Tech Carilion Research Institute (VTCRI) collaborating with the CURE Hospital in Addis Ababa. The study was reviewed and approved by the Ethiopia Ministry of Health and the institutional review boards of MUSC and VTCRI (Footnote 1 - to state that there was no established local or national institutional review board process in Ethiopia at the time we initiated the project.) The CURE Ethiopia Children's Hospital in Addis Ababa is a 36-bed hospital established in 2007 by CURE International (www. cure.org) with the primary medical intervention focused on surgical subspecialty care to children with motor disabilities, often followed by rehabilitation therapy services. CURE Hospital is accredited by College of Surgeons of East, Central and Southern Africa (based in the UK), and its staff includes western-trained surgeons and Ethiopian therapists, nurses and other allied healthcare providers. We note that CIMT is not a treatment designed for children undergoing orthopaedic procedures per se, but CURE recognized the potential added value of this EBP for its paediatric population, during both pre-surgery and post-surgery periods. In Ethiopia, as in other sub-Saharan countries, hospitals often identify a high-priority funding need (e.g. orthopaedic surgeries and leprosy treatment), and then, after establishing trust and learning about local needs, they expand to address a wider array of diagnoses and therapies, often serving as a central hub for other local hospitals and clinical practices.

Our research processes started when a medical director of the CURE Hospital in Addis Ababa contacted us, faculty members at MUSC and VTCRI, requesting a workshop on CIMT for CURE therapists and staff. After competitively securing funds from the Medical University of South Carolina Center for Global Health, we corresponded frequently with CURE Hospital's medical director and lead therapist to plan specific details for training, identifying scope of training, who would be invited, and what therapy and educational materials we would need. We determined that we could readily expand to include therapists from all other major hospitals treating children with $\mathrm{CP}$, as well as the CURE Hospital therapists and assistants. Figure 1 summarizes the steps we took to develop educational 


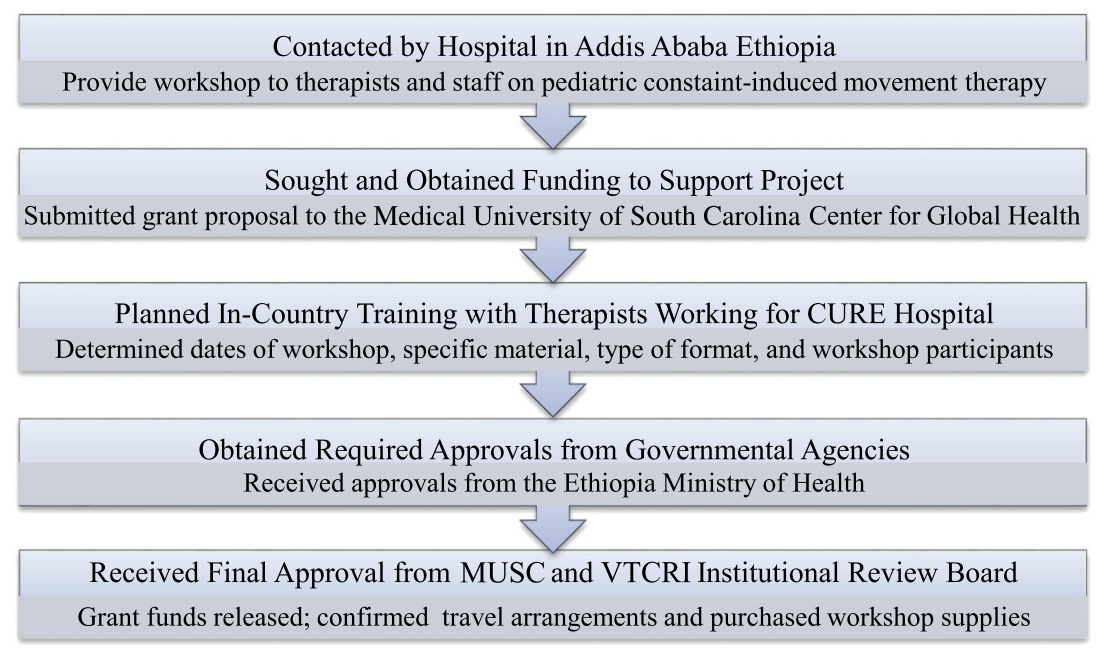

Figure 1. The process of development of an evidenced-based workshop in Ethiopia

content, instructional delivery modes and evaluation for EBP training.

This process or action plan is highly compatible with the $5 \mathrm{~A}$ 's of behaviour change - namely ask, assist, arrange, access and advise - that Estabrooks and Glasgow (2006) proposed. We selected the $5 \mathrm{~A}$ model because it had been successful already in guiding three notable trials of implementation of clinic-based physical activity interventions (Petrella et al., 2003; Ackermann et al., 2005; Pinto et al., 2005) and because the senior author of this model is a colleague and co-directs (with one of the authors of this paper) the Implementation Science track in a new $\mathrm{PhD}$ programme in translational science. We sought the advice of and interacted with the in-country partners by asking relevant questions. We then assisted personnel within CURE Hospital to identify potential barriers to the successful training and then implementation of P-CIMT (e.g. many children with $\mathrm{CP}$ will receive care from multiple institutions, so including therapists at all local hospitals would be vital for long-term success). We then developed and arranged a specific action plan with measurable goals and outcomes. Early on, we agreed that that we needed to assess our plan in a way that connects to our first steps of asking for advice and assistance from primary stakeholders. We saw this multilevel-system approach as an ongoing and dynamic process that required frequent adjustment of our actions before, during and after training. Figure 2

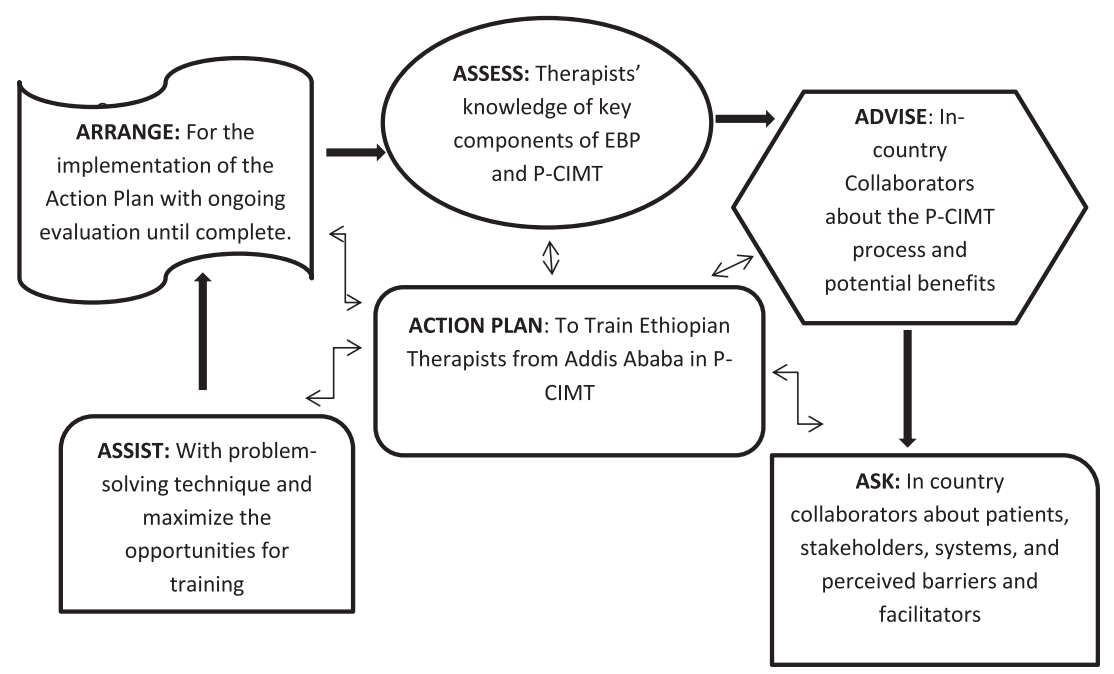

Figure 2. The $5 A^{\prime}$ 's of planning and enacting paediatric constraint-induced movement therapy (P-CIMT) training for therapists in Addis Ababa, Ethiopia, with examples 
illustrates the cyclical model we used to develop and implement the Ethiopia CIMT workshop, adapted from 5A's model (Estabrooks and Glasgow, 2006) paper.

\section{Therapists/workshop participant survey}

After conducting a baseline self-report of the therapists' current practices and knowledge about CIMT, we finalized a tool to assess workshop participants' knowledge of the critical elements of CIMT, the EBP being taught, and conducted in-person observations of therapists' skills during practice sessions with children. The tool focused on P-CIMT core elements that we had explicitly taught, demonstrated and discussed and that informed the therapist's joint goal setting with the parents and child, based on results from systematic baseline assessment of each child. Training included assessment methods, how to collaborate with and engage parents, the value of measuring changes during and after delivery of CIMT and potential challenges that require monitoring throughout CIMT implementation.

\section{Results}

\section{Engagement levels of workshop participants}

A total of ten physical therapists, one occupational therapist and one therapist assistant staff from five hospitals in Addis Ababa participated and completed the entire training workshop. CURE Hospital's lead therapist recruited all attendees (each provided therapy to children with neuromotor disabilities). We provided all-day training for 5 consecutive days at the CURE Hospital and visited two other hospital settings during the week, including the primary, large state-supported facility.

Workshop attendees included eight male physical therapists, two female physical therapists, one female occupational therapist and one female staff assistant who helped with patient care. On some days, we had as many as 17 attendees, including some administrators and other therapists who were not able to attend all 5 days. This variation in attendance likely occurred because approval for the training we offered took place at the government level, but we had not received strong or official pre-approval from the other four hospitals (i.e. outside the CURE sponsor). We did, however, observe high levels of interest and later support shortly after the participating therapists relayed new information to their administrators. Initially, each workshop participant affirmed that he or she could readily identify specific children they currently were treating who had asymmetric motor impairment and thus met the clinical eligibility criteria for CIMT. Workshop participants, with the exception of two, were fluent in speaking and reading English, although the primary language was Amharic for ten and Spanish or English each for one.

\section{Incentives and remuneration for workshop participants}

Attendees received training without paying fees, and we remunerated them from grant funds for their time lost from work, a customary practice in Addis Ababa and endorsed by CURE Hospital that recommended amounts based on current salaries (approximately \$US10 per day). CURE Hospital provided hot lunches and "tea times" in the morning and the afternoon. We gave therapists the Handbook of Pediatric Constraint-Induced Movement Therapy (Ramey et al., 2013), valued at $\$ 126$ each, along with copies of print and online training materials we used. Informally, many of the therapists received evening transportation services at no cost from CURE, and several socialization activities occurred after training sessions. We offered additional incentives for therapists who completed the workshop and then implemented the jointly adapted form of CIMT and submitted systematic documentation of therapy. This compensation covered additional time beyond their usual work hours to complete forms and communicate with us. We funded these activities at approximately \$US18 for completing the therapy log over the course of a month and \$US20 for completing detailed post-therapy summary assessments.

\section{Content of training workshop}

The 1-week training workshop included didactic educational sessions on the importance of EBP and the research findings supporting the use of CIMT for children (infancy through young adulthood) with asymmetric motor function. Didactic sessions occurred in the morning, with daily review of the prior day's key information and an opportunity to seek clarification before continuing with new material. From days 2 through 5, we included hands-on demonstration and learning opportunities with local children with hemiparesis. From the beginning, we actively sought contributions from the therapists about their 
current practices, their patient population and their own ideas about which features of CIMT would be easy to implement and which would benefit from adaptation to their situations. As we (the authors of this article and workshop faculty) learned about that, the therapists' beginning knowledge about EBPs and the relevant cultural practices and resource issues, we adapted the next day's materials in our nightly review and planning sessions (lasting about $2 \mathrm{~h}$ each).

We adapted our materials, for example when we learned that the predominant therapeutic modality used in children with $\mathrm{CP}$ was passive range of motion, combined with allowing the natural course of a child's development to occur. We then realized that our operative assumption that families would actively pursue therapy services to increase functional skills and would expect to learn new techniques to help their children at home and in school or community settings needed to be reframed. That is, working with the therapists, we explored whether these new approaches were likely to be well received (the answer was yes) and how best to communicate this with other healthcare professionals, parents and children themselves. Our finding supported Donald et al.'s (2014) observations about clinical management practices for CP in Africa. Of all the materials we used, we found that the training videotapes we showed, repeatedly, produced the highest level of excitement about the potential of children to change, rapidly and in functionally important ways. We used videotapes that showed the same children over the course of a month of CIMT treatment as well as several children who received CIMT over several consecutive years in their lives. The visual impact was high, and many therapists wanted to show these to others. In retrospect, seeing how powerful these video case examples were, compared with standard graphics displaying effect size and magnitude of change on standardized tools (tools that were not in use in Addis Ababa at that time), we would have included even more of these as part of the formal training curriculum. The formal sessions focused on the centrality of five essential components of CIMT: (1) use of an appropriate constraint for the child's less-impaired upper extremity; (2) delivery of high concentrated dosages of therapy (at least $2 \mathrm{~h}$ per day for a minimum of 2 weeks); (3) use of systematic shaping and reinforcement techniques, plus practice of new skills; (4) delivery of therapy within the child's natural environments (e.g. home or school); and (5) development of a transition (post-therapy) planning package that included bimanual functional activities.

We closely partnered these elements with hands-on sessions involving 18 Ethiopian children (all brought in by their parents; none were in-patients at hospitals) with unilateral motor impairment recruited by the CURE lead therapist. The children varied widely in age and severity of impairments, and provided an ideal opportunity to operationalize the content of the didactic sessions and for us to model the types of adaptations and clinical decision-making needed to develop an individualized therapy plan that incorporated each of the five core elements. Based on full assessments of all the children, a few were judged not to be good candidates for this form of EBP, and were able to discuss the reasons for this conclusion. Most of the children were ideal matches for CIMT, and we invited X to return on subsequent days with their parents (note: these children were among those who later received a longer course of CIMT). Workshop participants first observed and then individually constructed different forms of constraints, including special full arm-to-fingers casts and arm and hand splints/orthotics, with the new materials we brought (these differed from those in use locally). Figure 3 shows some of the highlights of each training day.

As a team, the therapists and faculty designed an adapted protocol for CIMT that seemed feasible, culturally relevant and consonant with core elements of EBP. Table I shows the adaptations made and compares these with some of the protocols that were used in the published clinical trials (DeLuca et al., 2006; Case-Smith et al., 2012; DeLuca et al., 2012; Coker-Bolt et al., 2013) that formed the basis for the type of CIMT we taught. All of these essential elements (active ingredients) remained in the final Ethiopian adaptation.

\section{Therapists/workshop participant survey}

We administered a formal test on the fifth (last) day. Therapists knew in advance about the post-training exam, and that the goal was to ensure learning and identify potential areas for follow-up, if needed. We also asked for feedback about the workshop methods and our instruction and solicited ideas for future improvements. All workshop participants who completed the workshop completed the postworkshop survey $(n=12)$. Each participant could accurately name and define the five essential components of CIMT. In addition, all 


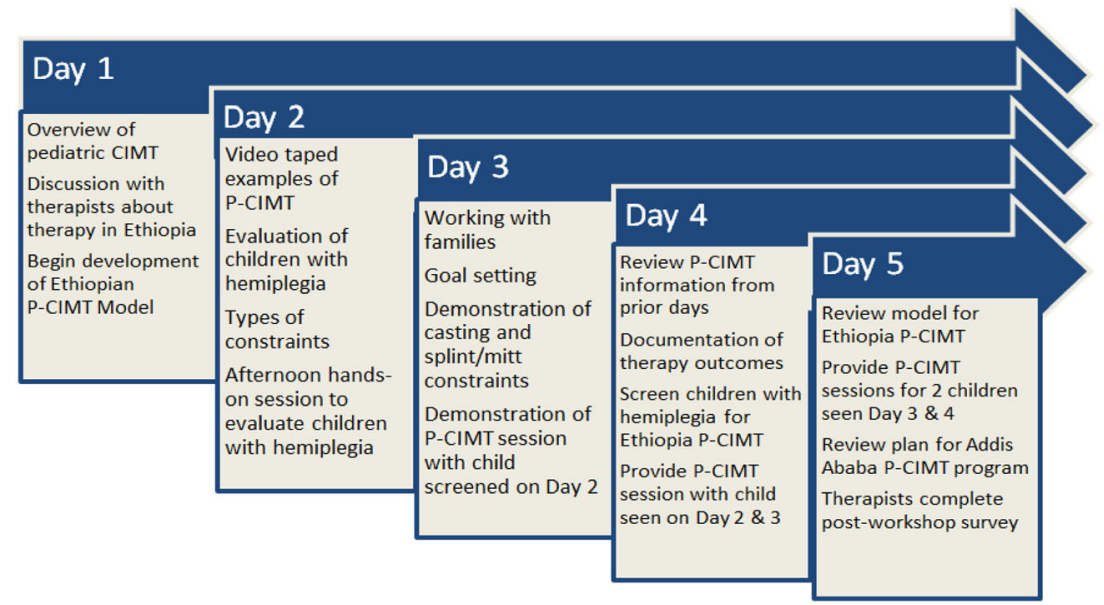

Figure 3. Description of educational sessions for 5-day workshop on paediatric constraint-induced movement therapy (P-CIMT) in Addis Ababa, Ethiopia

Table I. Ethiopian P-CIMT model created in collaboration with therapists who completed workshop training

Five essential components of P-CIMT (cf. Ramey et al., 2013) Signature or traditional P-CIMT protocol Ethiopian model of adapted P-CIMT

1. Constraint of the less or unimpaired upper extremity

2. High dosage (likely minimum threshold $=2$-h sessions per day for 5 days per week)

3. Use of shaping techniques and repetitive practice with task variation

4. Learning functional skills in natural and diverse settings

5. Transition (post-therapy) planning for maintenance of gains
Constraint of the less or unimpaired upper extremity using cast for majority of waking hours and during active treatment

High dosage of therapy in concentrated period of time involving active treatment for a minimum of $3 \mathrm{~h}$ a day for 5 days per week for multiple weeks

Use of shaping techniques to review, extend, practice and refine skills that uses formal operant learning techniques with immediate feedback and reinforcement with all treatment sessions Learning functional skills in natural and diverse settings (i.e. treatment is in these settings)

Post-therapy planning to promote functional bilateral and unilateral upper extremity development and continued practice of new skills with more impaired upper extremity
Constraint using full-arm cast (plaster or fibreglass) or resting-hand splint with puppet or mitt used during therapy and worn at home during day

Therapist creates a partnership where the therapist provides $1 \mathrm{~h}$ of therapy and the parent provides $1 \mathrm{~h}$ of therapy (2-h total therapy) for 5 days a

week for 4 weeks in a row (dosage $=40 \mathrm{~h})$ Use of shaping techniques to review, extend, practice and refine skills as an active component of treatment

Treatment in a clinic setting where focus is on doing the types of things children do at home and in everyday life; backpack with toys sent home after each session to encourage practice at home with family Post-therapy planning to allow the child and parents to continue to work on new skills and not lose the benefits of the month of high-intensity P-CIMT

P-CIMT = paediatric constraint-induced movement therapy.

identified specific functional goal areas and overall skills that CIMT could improve significantly. The area of greatest difficulty, however, concerned therapists' ability to identify important elements of a discharge programme following CIMT with $75 \%$ not able to do this with sufficient specificity. We then realized that we may not have dedicated enough time to this topic, especially given the knowledge we received that the idea of a "home programme" was a new concept for the therapists and the families. Some therapists reported that limited family 
resources would be a barrier to children completing therapy activities in the home. The participants provided generally highly favourable feedback on the workshop, the value of what they had been learned, the benefits of providing P-CIMT in Addis Ababa, Ethiopia, and potential challenges to providing $\mathrm{P}-\mathrm{CIMT}$ in their practice settings (Table II).

\section{Discussion}

We successfully implemented the jointly planned weeklong training session focused on implementing a relatively new form of EBP, namely CIMT for children with hemiparesis, in a sub-Saharan African city. Our work helps to provide practical guidance so that
Donald et al.'s (2014) recommendation for assessing the management of children with $\mathrm{CP}$ in Africa can be realized - that is, to apply new models for provider training in which visiting specialists provide valuable, focused training with active engagement of Africans who, in turn, can expand and train others in their country. We benefitted from and highly endorse the use of a multilevel-system approach in which we engaged stakeholders (organizations/administrators and clinics/departments) in each stage of planning and delivering of educational materials to the frontline therapists responsible for translating current evidence into practice in a region of the world with limited resources. We designed, and then made daily adaptations, for a week-long curriculum that balanced lectures,

Table II. Examples of post-training feedback from therapists (with small modifications to protect anonymity of workshop of participants)

Participants comments after completing workshop

Overall workshop feedback

Benefits of P-CIMT for children in Ethiopia

Challenges of providing P-CIMT in Addis Ababa, Ethiopia

Improvements seen in child following P-CIMT

Overall impression of P-CIMT
"It was a very good workshop. This will help us to provide more therapeutic benefits to our patients. You showed us a new way of treating weakness and disability that we can now use."

"This therapy should be carried on at other hospitals, too. This approach will greatly change the way our children live in Ethiopia" "I have learned many things from the workshop and especially how practical P-CIMT can be. I am hopeful this therapy will be much more effective than what we currently have been doing in Ethiopia." "I like what the idea of what P-CIMT means; (In our culture), in a right handed person, if the hand right hand is injured, the person is unlikely to use his left hand. We can provide therapy (CIMT) to help him use his right hand again."

"We loved the workshop. It would be good to provide us with some materials to provide P-CIMT and we would also love reading materials and more training"

"To get great and functional progression within a limited period of time." "Change the life expectation of the child to his family and our society."

"The parents will develop good hope and better life (less stigma)."

"Improvement of functional activities in a short period."

"It will help the patients use or try to use their weak UE and to help their children be more active, independent."

"Better function at home and (ability to) go to school, since children have to be able to self-care to go to school here."

"Limitation of resources"

"Working areas may not be suitable for the therapist and family"

"Few human resources (therapist)"

"Socioeconomic structure; socio-economic status of the family"

Therapists Comments After Implementing P-CIMT with a Child

"His fine motor skills are improved, like he can pick up small objects using his thumb and index finger and release objects easily"

"I would like to apply CIMT for another child too. I did it for a child who has cerebral palsy and she improved a lot. This is wonderful treatment."

"Home exercises are very helpful, the child only spent $2 \mathrm{~h}$ a day in therapy but he can do all the exercises at home during lots of different times and in different activities."

$\mathrm{P}$-CIMT $=$ paediatric constraint-induced movement therapy. 
discussions, video and in-person demonstrations, and hands-on practical learning experiences to convey core concepts and specifically designated therapy skills. Throughout the workshop, the jointly embraced goal was to develop and then test a clinical protocol that the therapists embraced as culturally and practically well-matched for their patients and clinical practices. Although we did not formally seek to promote team building per se, in fact this occurred among the therapists (some of whom already knew one another and/or had attended college together), setting the stage for later sharing of available resources and potentially creating a network of providers. Figure 4 captures the levels of engagement and the topics we considered throughout the partnership and training week. We know that local as well as international relationships and alignment issues can be highly facilitative and functionally exist within the system models where implementation occurs and the cultural/social-environmental context matters (Estabrooks and Glasgow, 2006; Dizon et al., 2012).

Our study affirms the value and centrality of forming strong partnerships at the outset, even prior to developing educational materials. We sought to evaluate each of our educational sessions and make adjustments in the remaining sessions. To what extent the high levels of receptivity and enthusiasm among both administrators and therapists provide a close-to-ideal situation is something that we cannot appraise formally. We reflect that one member of our team (Patty Coker-Bolt) had years of experience bringing EBP in other areas to other African nations; her comfort and skill in communicating, socializing and observing assisted the other faculty (Sharon Ramey and Stephanie DeLuca) who were new to sub-Saharan Africa, but highly experienced in conducting intensive CIMT training in the United States. We agree with Curran et al. (2012) about the perceived value of systematic, frequent communication with partners to increase the success of adapting clinical strategies, including documentation of outcomes, to the realities of the local practice and cultural context. We were grateful for the constructive nature of the feedback we received from workshop participants about the practical value of the workshop, and we developed a long list of extra things to do in the future. We preserved the training curriculum and assessment tools so that this model could be replicated elsewhere (footnote that will encourage readers to request copies of training materials at no cost), with improvements and adaptation to other partners. We conclude that keys to our success were high daily attendance, because the learning built is upon prior experiences and contained ongoing review. Without incentives and remuneration for the therapists' time, we think we could not have achieved the level of consistent engagement we realized.

As we stated earlier, the local therapists expressed a strong desire to learn and implement EBP and related openly their frustration with use of therapy interventions that focused solely on passive movement, manipulation techniques and contracture management using casting and did not yield observable functional benefits. Therapists enjoyed the use of active and collaborative learning strategies that allowed them to be a part of developing a new Ethiopian model of P-CIMT based on current research. Therapists played a large role in ensuring the model was culturally sensitive and could be implemented within the therapy practice milieu of Addis Ababa. For example, in Ethiopia, a person uses the right and left hands differentially for specific daily

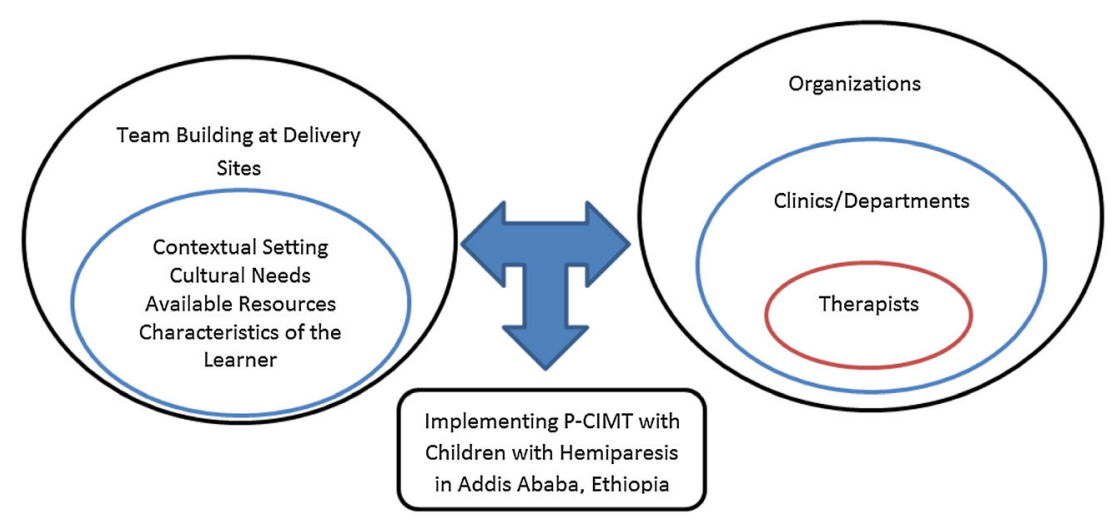

Figure 4. Evidenced-based training model to advance use of paediatric constraint-induced movement therapy (P-CIMT) in sub-Saharan Africa 
tasks (i.e. right hand for clean activities such as feeding and left hand for personal hygiene and other tasks that could involve exposure to germs and unclean materials); accordingly, targeted goals for therapy were developed that took into account these differential functional activities designated as important within the Ethiopian culture. In addition, the therapists suggested adapted models for task completion and assessment to meet the specific needs of their culture (e.g. use of culturally specific hand gestures and lack of utensil use in most eating activities).

Although the therapists and the facilities responded well to the training and were able to implement the new Ethiopian CIMT model with children evaluated at the workshop, we learned several important lessons that should be considered when planning EBP workshops for therapists in developing countries. Chief among these are the following: (1) Strong consideration should be made to the type of communication available to in-country collaborators before, during and after the planned workshop sessions. Consistent communication systems, such as phone, e-mail and video conferencing/telecommunication (e.g. Skype) have a large impact on timely, effective translation of information to all workshop trainees. We lacked this, unfortunately, with many of our therapists in Addis Ababa, and this led to some missed opportunities for timely feedback and data gathering. (2) We encountered complications and delays in shipping crucial therapy materials, because in part due of fees and taxes placed on medical supplies by governmental agencies. (3) We had not sufficiently developed a long-term plan for the partnership or engaged the local colleges that trained therapists, to contribute to a more sustainable infrastructure.

\section{Limitations}

The partnership presented in this article was limited to the outcomes of training paediatric therapists in subSaharan Africa and does not include data on how the new Ethiopian model of CIMT was used in practice. We believe this paper provides valuable information about how to successfully train therapists in low resource countries about EBPs. Formation of key partnerships is important for the training process and promoting the highest outcomes from training. Understanding how to most effectively implement training about EBPs builds the foundation for evidence-based interventions to be applied within these environments. A second article will provide outcome data on the application of the new Ethiopian model of CIMT for children with hemiplegic CP in Addis Ababa. This article will show how therapists who participated in the training workshop were able to implement the new Ethiopian adapted model of CIMT successfully in children with unilateral motor weakness.

\section{Conclusion}

The data presented here support the establishment of a successful training partnership, with evidence that participating therapists learned new skills, effectively demonstrated their application in a clinical context and expressed enthusiasm for the final locally developed, culturally relevant model of EBP that resulted from the partnership. During our visit and after, we learned of other international partnerships (varying in their resources, longevity and success to date) that appeared to align well with the goals of our partnership; thus, we hope to expand and ensure that our colleagues in Ethiopia will become experts and leaders who receive local and international support for implementing CIMT and other forms of EBP, far beyond the focus of our project.

\section{Acknowledgements}

This work is supported by a pilot projects grant from the Medical University of South Carolina Center for Global Health.

\section{REFERENCES}

Ackermann RT, Deyo RA, LoGerfo JP (2005). Prompting primary providers to increase community exercise referrals for older adults: a randomized trial. Journal of the American Geriatrics Society 53: 283-289.

Case-Smith J, DeLuca SC, Stevenson R, Ramey SL (2012). Multicenter randomized controlled trial of pediatric constraint-induced movement therapy: 6-month follow up. American Journal of Occupational Therapy 66(1): $12-23$.

Coker-Bolt P, Reidy T, Nabor E (2013). Alternative pediatric CIMT: understanding the how and why of clinical variations in pediatric CIMT. In Ramey SL, Coker-Bolt P, DeLuca SC (eds). A Handbook of Pediatric Constraint-induced Movement Therapy (P-CIMT): A Guide for Occupational and Physical Therapists, Researchers, and Clinicians. Bethesda, MD: The American Occupational Therapy Association Press. 
Curran G, Bauer B, Mittman B, Pyne J, Stetler C (2012). Effectiveness-implementation hybrid designs: combining elements of clinical effectiveness and implementation research to enhance public health impact. Medical Care 50(3): 217-226.

Dawes M, Summerskill W, Glasziou P, Cartabellotta A, Martin J, Hopayian K, Porzsolt, F, Burls A, Osborne J (2005). Second International Conference of Evidencebased Health Care Teachers and Developers. BMC Medical Education 5: 1-7.

DeLuca SC, Echols K, Law CR, Ramey SL (2006). Intensive constraint-induced therapy for children with cerebral palsy: randomized, controlled, crossover trial. Journal of Child Neurology 21(11): 931-938.

DeLuca SC, Case-Smith J, Stevenson R, Ramey SL (2012). Constraint-induced movement therapy (CIMT) for young children with cerebral palsy: effects of therapeutic dosage. Journal of Pediatric Rehabilitation and Medicine 5(2): 133-142.

Dizon J, Grimmer-Somers K, Kumar S (2012). Current evidence on evidence-based practice training in allied health: a systematic review of literature. International Journal of Evidenced Based Healthcare 10: 347-360.

Dizon J, Dizon J, Regino J, Gabriel A (2014). Evidencebased practice training for health professionals in the Philippines. Advances in Medical Education and Practice, 5: 89-94.

Donald K, Kakooza A, Wammanda R, Mallewa M, Samia P, Babakir H, Bearden D, Majnemer A, Fehlling D, Shevell M, Chugani H, Wilmshurst J (2014). Pediatric cerebral palsy: where are we? Journal of Child Neurology 10: 1-9.

Estabrooks P, Glasgow R (2006). Translating effective clinic-based physical activity interventions into practice.
American Journal of Preventative Medicine 31: S45-S52.

Idro R, Newton C, Kiguli S, Kakooza-Mwesige A (2010). Child neurology practice and neurological disorders in East Africa. Journal of Child Neurology 25(4): 518-524.

Grimshaw J, Eccles M, Tetroe J (2012). Implementing clinical guidelines: current evidence and future implications. Journal of Continuing Education in Health Professions 24: 31-37.

Novak I, McIntyre S, Morgan C, Campbell L, Dark L, Morton N, Stumbles E, Wilson SA, Goldsmith S (2013). A systematic review of interventions for children with cerebral palsy: state of evidence. Developmental Medicine and Child Neurology 55(10): 885-910.

Petrella R, Koval J, Cunningham D, Paterson D (2003). Can primary care doctors prescribe exercise to improve fitness. American Journal of Preventative Medicine 3 (24): 316-322.

Pinto BM, Goldstein MG, Ashba J, Sciamanna C, Jette A (2005). Randomized controlled trial of physical activity counseling for older primary care patients. American Journal of Preventative Medicine 29: 247-255.

Ramey SL, Coker-Bolt P, DeLuca SC (2013). A Handbook of Pediatric Constraint-induced Movement Therapy (PCIMT): A Guide for Occupational and Physical Therapists, Researchers, and Clinicians. Bethesda, MD: The American Occupational Therapy Association Press.

Schreiber J, Stern P (2005). A review of the literature on evidence-based practice in physical therapy. Internet Journal of Allied Health Sciences and Practice 3: 1-10.

World Health Organization (2011). International Classification of Impairments, Disability and Handicaps (ICIDH). WHO Press, World Health Organization, Geneva, Switzerland, ISBN 8799241564182. 